\title{
ORIGINAL ARTICLE \\ Gluteal blood flow and oxygenation during electrical stimulation-induced muscle activation versus pressure relief movements in wheelchair users with a spinal cord injury
}

\author{
CAJ Smit ${ }^{1}$, M Zwinkels ${ }^{2}$, T van Dijk ${ }^{2}, S$ de Groot ${ }^{1,3}$, JM Stolwijk-Swuste ${ }^{1}$ and TWJ Janssen ${ }^{1,2}$
}

Background: Prolonged high ischial tuberosities pressure (IT pressure), decreased regional blood flow (BF) and oxygenation (\%SO2) are risk factors for developing pressure ulcers (PUs) in patients with spinal cord injury (SCl). Electrical stimulation (ES)-induced gluteal and hamstring muscle activation may improve pressure distribution by changing the shape of the buttocks while sitting and also increase $\mathrm{BF}$ and \%SO2.

Objective: To compare acute effects of ES-induced gluteal and hamstring muscle activation with pressure relief movements (PRMs) on IT pressure, BF and \%SO2.

Participants and methods: Twelve men with SCl performed PRMs - push-ups, bending forward and leaning sideward - and received surface ES $(87 \pm 19 \mathrm{~mA})$ to the gluteal and hamstring muscles while sitting in their wheelchair. Ischial tuberosities pressure was measured using a pressure mapping system; (sub)cutaneous BF and \%SO2 were measured using reflection spectroscopy and laser Doppler, respectively.

Results: Compared with rest $(156 \pm 26 \mathrm{~mm} \mathrm{Hg})$, IT pressure was significantly lower during all other conditions (push-ups $19 \pm 44$; bending forward $56 \pm 33$; leaning sideward $44 \pm 38$; ES $67 \pm 45 \mathrm{~mm} \mathrm{Hg}$ ). For the whole group, all PRMs significantly augmented BF $(+39$ to $-96 \%)$ and \%SO2 ( +6.0 to $-7.9 \%$-point), whereas ES-induced muscle activation did only for peak BF. In all, $63 \%$ of the participants showed an increased BF (average $52 \%$ ) with ES.

Conclusion: PRMs acutely reduced IT pressure and improved oxygenation and $\mathrm{BF}$ in $\mathrm{SCl}$. The currently used ES method cannot replace PRMs, but it may be used additionally. ES-induced muscle activation is not as effective for acute pressure relief, but the frequency of stimulation is much higher than the performance of PRMs and can therefore be more effective in the long term.

Spinal Cord (2013) 51, 694-699; doi:10.1038/sc.2013.66; published online 2 July 2013

Keywords: spinal cord injury; pressure ulcers; electrical stimulation; sitting pressure; oxygenation; blood flow

\section{INTRODUCTION}

Pressure ulcers (PUs) are the most prevalent secondary complications in individuals with a spinal cord injury (SCI). Throughout their life, up to $80 \%$ of people with an SCI suffer from a PU, ${ }^{1,2}$ leading to radical consequences such as decreased mobility and independence, delayed rehabilitation, and exclusion from social activities, ${ }^{2,3}$ and it has a tremendous impact on the individual's physical condition. ${ }^{3,4}$ The consequences also result in high costs for the community. ${ }^{4,5}$

Shifting posture regularly, on average eight times an hour, prevents healthy individuals from developing PUs, as subcutaneous oxygenation increases with each posture adjustment. ${ }^{5}$ Preventive measures like pressure relief movements (PRMs) and pressure distributing cushions are passive methods and are not adequate enough, as PUs still occur. Besides, although PRMs such as bending forward, leaning sideward and/or lifting improve blood circulation in compromised tissue, they require good upper-limb strength and generate high pressure on the gleno-humeral joint, possibly resulting in shoulder injuries. ${ }^{6}$ In addition, the person must be well motivated to continue lifting eight times every hour. Moreover, tissue properties do not improve.
Recent research showed that electrical stimulation (ES) of the tuber area in individuals with an SCI had a direct positive effect on interface pressure distribution, blood flow (BF) and muscle size. ${ }^{7-11}$ Levine et al. ${ }^{8,9}$ used surface ES in the gluteal muscles of individuals with an SCI and found a changed buttock tissue shape ${ }^{8}$ and increased muscle $\mathrm{BF}^{9}{ }^{9}$ Ferguson $e t$ al. ${ }^{7}$ stimulated the quadriceps muscles and found a decrease in interface pressure as well. Smit et al. ${ }^{12}$ found that ES-induced gluteal and hamstring muscle activation reduces IT pressure and, in a 3-h stimulation period with a 1:4 s on-off duty cycle, does not cause muscle fatigue. Bogie et al. ${ }^{1}$ examined the longterm effects of ES, using implanted electrodes in the gluteal muscles to electrically stimulate the muscles. This long-term study found increased gluteal muscle thickness and increased $\mathrm{BF}$, together with a decrease in interface pressure. A more practical intervention was recently introduced in a study by Smit et al.,12 describing the effects of a noninvasive ES method on interface pressure distribution, using electrostimulation-shorts (ES-shorts).

The positive effect of ES on interface pressure as well as the positive effect of shifting posture on oxygenation has been demonstrated ${ }^{13,14}$

${ }^{1}$ Amsterdam Rehabilitation Research Center, Reade, Amsterdam, The Netherlands; ${ }^{2}$ Research Institute MOVE, Faculty of Human Movement Sciences, VU University, Amsterdam, The Netherlands and ${ }^{3}$ University of Groningen, University Medical Center Groningen, Center for Human Movement Sciences, Groningen, The Netherlands Correspondence: Dr CAJ Smit, Spinal Cord Injury Ward, Amsterdam Rehabilitation Research Center, Reade, Location Overtoom, PO Box 58271,1040 HG Amsterdam, The Netherlands.

E-mail: c.smit@reade.nl

Received 27 March 2013; revised 21 May 2013; accepted 24 May 2013; published online 2 July 2013 
However, there is no extensive literature describing the effect of ES on ischial oxygenation, nor has the effect of ES been compared with the effect of PRMs or the relationship between IT pressure and oxygenation and BF been described.

The present study therefore aimed to answer the following questions:

(1) What are the acute effects of ES-induced gluteal and hamstring muscle activation on IT pressure, BF and oxygenation, compared with three pressure relief movements?

(2) Is there a relationship between IT (sitting) pressure and oxygenation or BF?

We hypothesized that ES and PRMs would both decrease IT pressure and increase $\mathrm{BF}$ and oxygenation (\%SO2) and that a negative relationship exists between interface pressure and oxygenation or BF.

\section{MATERIALS AND METHODS}

\section{Participants}

Twelve men with SCI, having a complete or incomplete upper motor neuron lesion (ASIA A or B), aged between 18 and 60 years, were recruited for this study, which was approved by the local medical ethics committee. Gluteal and hamstring muscles, skin under the buttocks and spinal reflexes had to be intact, which were evaluated for all participants by a single physician. Excluded were individuals with a flaccid paralysis and areflexia, a history of severe autonomic dysreflexia, current IT PUs, severe cognitive or communicative disorders, intolerance for ES or any other contraindication for ES. Participants provided written informed consent. Participants' characteristics are described in Tables 1 and 2 .

\section{Study design}

This study was performed in a Rehabilitation Research Centre. The process of taking measurements lasted for about $4 \mathrm{~h}$ per participant. Each participant used his or her own wheelchair with a daily-use cushion; most of them were

Table 1 Participants' group characteristics

\begin{tabular}{lc}
\hline & Mean \pm s.d. (range) \\
\hline Participants (M/F) & $12(12 / 0)$ \\
Age (years) & $38.1 \pm 12.8(26-52)$ \\
Lesion level & C3-C8 $(N=7)$ Th1-Th12 $(N=5)$ \\
AIS impairment classification & ASIA A: 9 ASIA B: 3 \\
Time since injury (months) & $173 \pm 93(85-503)$ \\
Body mass (kg) & $82.2 \pm 15.0(64-107)$ \\
\hline
\end{tabular}

Table 2 Participants' individual characteristics

Participant Gender M/F Age Lesion level AIS score Time since injury (years)

\begin{tabular}{lllllr}
\hline 1 & M & 41 & C5 & B & 8.1 \\
2 & M & 32 & C6 & B & 12.0 \\
3 & M & 28 & C7 & A & 7.2 \\
4 & M & 50 & C8 & B & 9.9 \\
5 & M & 38 & C6 & A & 10.0 \\
6 & M & 52 & C6 & A & 16.8 \\
7 & M & 37 & C5 & A & 13.2 \\
8 & M & 28 & T11 & A & 18.8 \\
9 & M & 41 & T10 & A & 41.9 \\
10 & M & 48 & C7 & A & 19.6 \\
11 & M & 36 & C4 & A & 15.8 \\
12 & M & 26 & T8 & A & 7.8 \\
\hline
\end{tabular}

aircushions ( $n=10$; ROHO, Vicair or Starlock); some used gel ( $n=2$; Jay2), with a force-sensitive array on top. Before taking measurements, a $1.0 \times 1.5 \mathrm{~cm}^{2}$ probe, $0.1-\mathrm{cm}$ thick, was attached under the left ischial tuberosity with surgical tape while the participant was sitting in his chair and bent over to the right. After attachment of the probe, it was connected to the oxygenation device. Participants were then asked to perform PRMs in a counterbalanced order: bending forward, leaning sideward (to the right side to release the probe) and push-ups, for as long as possible, for a maximum of $2 \mathrm{~min}$ (Figure 1). Before each measurement the participant had to sit and rest for $5 \mathrm{~min}$. Every measurement started with $30 \mathrm{~s}$ of rest to gain a baseline value of interface pressure and oxygenation. Thereafter, after a $30 \mathrm{~min}$ rest period, in which self-adhesive surface electrodes, two on each leg, were applied, both gluteal and hamstring muscles were activated by ES. Outcome variables including IT pressure, ischial oxygenation and BF were measured at rest, during all pressure relief conditions and during ES-induced gluteal and hamstring muscle activation.

\section{Interface pressure measurement}

Interface pressure distribution was measured using a pressure mapping device (a force-sensitive array, mFlex, Vista Medical Europe, Venlo, the Netherlands): a 2-mm-thick soft flex mat of $42 \times 42 \mathrm{~cm}^{2}$ consisting of 256 pressure sensors, placed between the cushion on the wheelchair and the buttocks of the participant. Before testing, it was calibrated between 0 and $200 \mathrm{~mm} \mathrm{Hg}$ according to the systems' calibration protocol.

The participant had to sit in a 'normal position': feet on the footrests, arms on the armrests (if any) or on the participant's lap and the lower back against the backrest of the wheelchair. Before commencement of the protocol, the individual had to sit in his wheelchair on the FSA for $5 \mathrm{~min}$ to allow the cushion to adapt to the participant's buttocks. Pressure data were recorded continuously (one per second). Both IT areas were defined as the $3 \times 3$ sensors with the highest pressure values. Mean pressure values of both IT areas were used for statistical analysis (see Figure 2).

\section{Oxygenation and circulation}

Oxygenation data were obtained using an Oxygen To See device (O2C, LEA Medizintechnik GmbH, Giessen, Germany). The rigid O2C probe can measure the oxygen saturation of hemoglobin $\left(\% \mathrm{SO}_{2}\right)$ at the venous end of the capillaries, the quantity of hemoglobin in the micro-blood vessels and the velocity of flow of the blood in the microcirculation using a noninvasive combination of reflection spectroscopy and the laser Doppler technique. According to the manufacturer's manual, $\mathrm{O} 2 \mathrm{C}$ reliably measures to a depth of $\sim 10 \mathrm{~mm} . \% \mathrm{SO} 2$ is used as an indicative measure for oxygenation in the muscle tissue. ${ }^{5}$ As soon as \% $\mathrm{SO} 2$ reached a constant value, the mean \% $\mathrm{SO} 2$ and mean $\mathrm{BF}$ and peak $\mathrm{BF}$ were calculated. To correlate oxygenation and $\mathrm{BF}$ with interface pressure data, both measurements were taken simultaneously.

\section{Electrical stimulation}

A stimulator (NeuroPro 8 channel, Axiobionics, Ann Arbor, MI, USA) was connected with two surface electrodes per leg positioned at the upper (proximal) part of the gluteal muscle above the sitting area, and one about halfway of the hamstring area, preventing the participant from sitting on electrodes or on the wires while being stimulated (Figure 3). ES was delivered at standard $150 \mathrm{~V}$, with $50 \mathrm{~Hz}$ (bi-phasically) to induce a (visible) tetanic contraction. Before stimulation, the current amplitude resulting in the best pressure reduction was determined for each participant by increasing the amplitude in steps of 5-10 $\mathrm{mA}$ to a maximum, without discomfort or excessive muscle contractions disturbing normal sitting. This amplitude was applied with a duty cycle of $1 \mathrm{~s}$ stimulation and $4 \mathrm{~s}$ rest for $3 \mathrm{~min}$. We did not train the participants with ES.

\section{Statistical analysis}

Values were described by mean values \pm s.d. A repeated-measures analysis of variance (ANOVA) was performed to compare oxygenation, BF and IT pressure between all conditions (rest, PRMs and ES). A post-hoc test was performed when a significant difference was found $(\alpha<0.05)$ to indicate which conditions differed from each other. The relationship between oxygenation and 
a

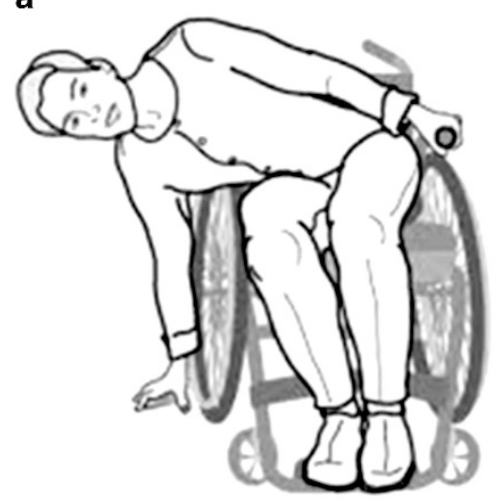

b

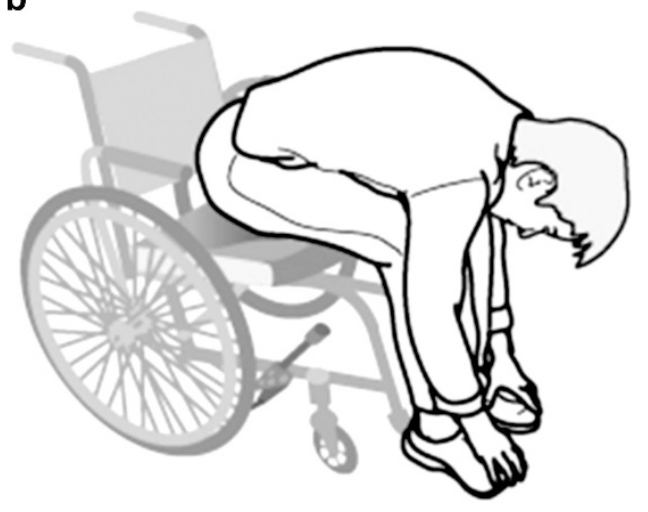

Figure 1 Examples of PRMs. (a) leaning sideward. (b) bending forward.

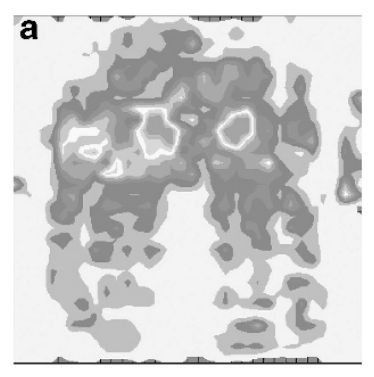

b

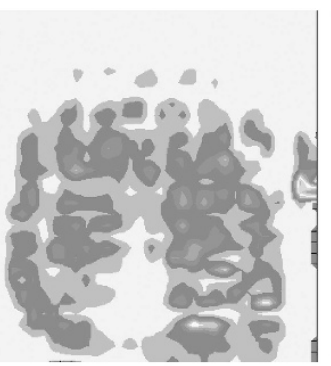

C

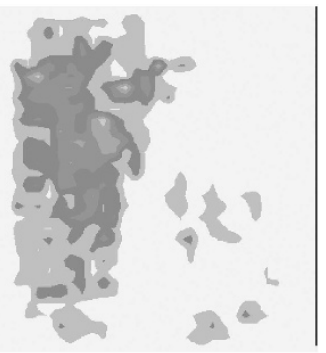

d

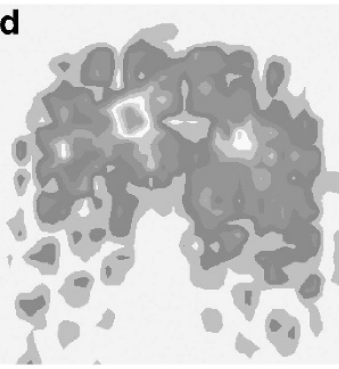

e

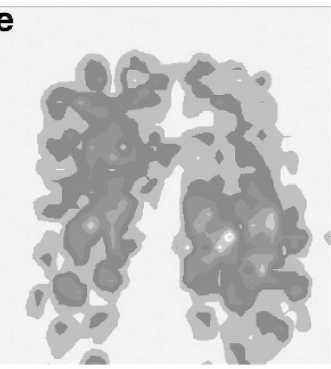

Figure 2 Raw interface pressure data of the mFLEX during rest (a), bending forward (b), leaning sideward (c), a stimulation period without (d) and with (e) ES-induced muscle contraction. Push-ups are left out, because they showed no interface pressure. Red color indicates high pressure and blue indicates low.
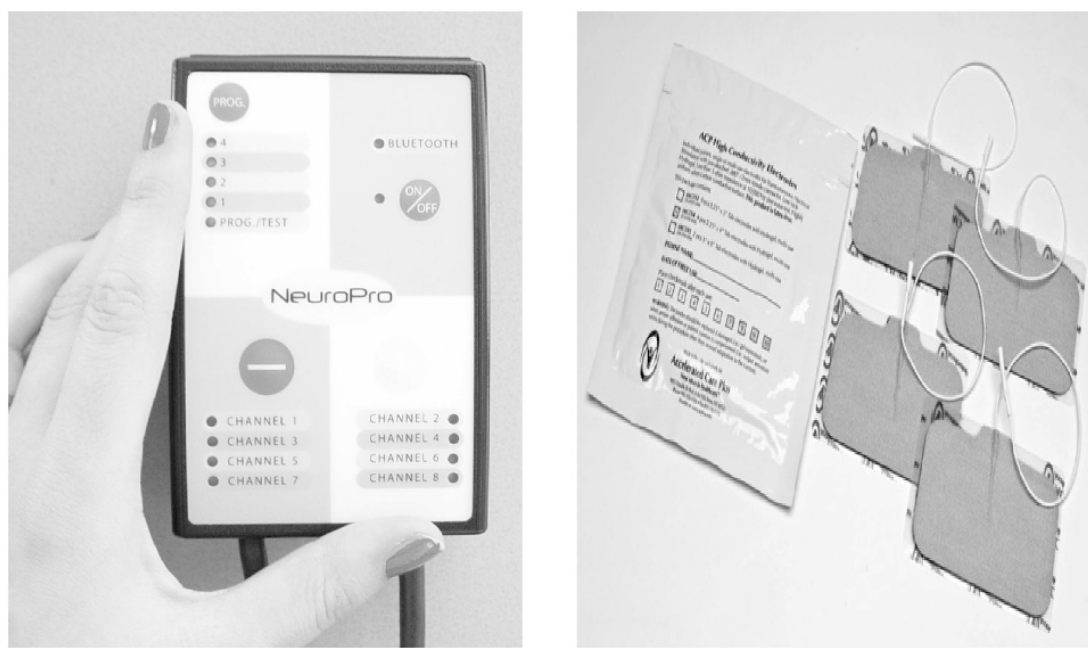

Figure 3 NeuroPro 8-channel electrostimulator, Axiobionics, Ann Arbor, MI, USA with self-adhesive surface electrodes. 
IT pressure and between (peak) BF and IT pressure was calculated by correlating mean changes between rest and other conditions (Pearson's correlation coefficient). Statistical analysis was performed using SPSS for Windows 17.0 (Chicago, IL, USA).

\section{RESULTS}

During this study, none of the participants developed skin problems due to the rigid $\mathrm{O} 2 \mathrm{C}$ probe or complaints of autonomic dysreflexia during PRMs, nor during ES-induced gluteal and hamstring muscle activation. The amplitude of ES varied between $55 \mathrm{~mA}$ and $125 \mathrm{~mA}$, with an average of $87 \mathrm{~mA}( \pm 18.5)$.

\section{IT pressure}

Compared with rest $(156 \pm 26 \mathrm{~mm} \mathrm{Hg})$, IT pressure was significantly lower during all other conditions (push-ups $19 \pm 44 \mathrm{~mm} \mathrm{Hg}$ $(P<0.001)$; bending forward $56 \pm 33 \mathrm{~mm} \mathrm{Hg}(P<0.001)$; and leaning sideward $44 \pm 38 \mathrm{~mm} \mathrm{Hg}(P<0.001))$. ES-induced gluteal and hamstring muscle activation reduced IT pressure to $67 \pm 45 \mathrm{~mm} \mathrm{Hg}$ $(\mathrm{p}=0.03)$. The post hoc test indicated no significant difference between PRM and ES conditions.

\section{Oxygenation}

Because of technical problems with the $\mathrm{O} 2 \mathrm{C}$, only the data of nine participants were reliable. Bending forward $(P=0.01)$, leaning sideward $(P=0.01)$ and push-ups $(P=0.01)$ significantly increased mean oxygenation compared with rest, whereas ES did not $(P=0.57)$. No significant difference was found between the different passive PRMs (Figure 4). A weak and nonsignificant correlation was found between mean oxygenation change and (mean) IT pressure change for the PRMs, whereas it was strong and significantly correlated for ES $(r=0.7$; Table 3$)$.

\section{Blood flow}

Compared with rest, bending forward $(P=0.02)$, leaning sideward $(P=0.03)$ and push-up $(P=0.02)$ increased the BF significantly. However, ES did not cause a significant change $(P=0.75)$ in mean BF. There was a significant difference in peak BF for ES $(P=0.007)$ and for bending forward $(P=0.006)$ compared with rest (Table 4). Change in BF was significantly lower with ES than with all PRMs, and a significantly larger increase was found for push-ups compared with leaning sideward, but not when compared with bending forward (Figure 5). A clear correlation was found between BF change and mean interface pressure change for all conditions, although it was only moderate for push-ups and for ES (Table 3). However, Figure 6

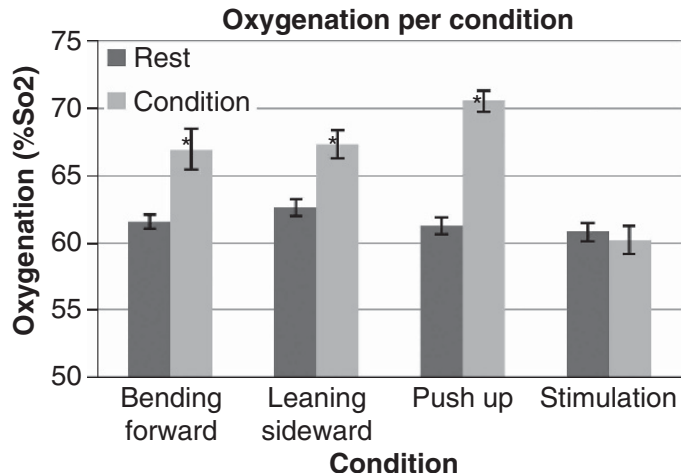

Figure 4 The mean IT oxygenation per condition compared with rest just before performing the action. *Significant increase in oxygenation compared with rest. indicates a certain relationship over time for two participants, as the $\mathrm{BF}$ increases directly after applying ES while the interface pressure decreases.

\section{DISCUSSION}

The present study showed that PRMs induced significant acute reduction in interface pressure and directly improved (sub)cutaneous oxygenation and BF in sitting wheelchair users with SCI. We hypothesized that ES-induced muscle activation would also increase BF and oxygenation; however, this study does not support that hypothesis. The relation between IT pressure and circulation showed that $\mathrm{BF}$ is influenced by IT pressure in some participants. ES increased $\mathrm{BF}$ and oxygenation in 8 of 12 participants (63\%) (Figure 6); however, ES did not change mean BF or oxygenation when analyzed over the whole group (Figure 5). ES and bending forward significantly increased peak BF. For ES this can be explained by the $1 \mathrm{~s}$-induced muscle contraction causing a peak in $\mathrm{BF}$, as well as a reduction in $\mathrm{BF}$ when the muscle relaxes again. Average values of BF are lower of

Table 3 Correlations between changes in interface pressure and oxygenation and interface pressure and blood flow

\begin{tabular}{|c|c|c|c|c|}
\hline \multirow[t]{2}{*}{ Correlation } & \multicolumn{2}{|c|}{$\begin{array}{c}\text { Oxygenation_interface } \\
\text { pressure }\end{array}$} & \multicolumn{2}{|c|}{$\begin{array}{c}\text { Blood flow-interface } \\
\text { pressure }\end{array}$} \\
\hline & r & $P$ & r & $P$ \\
\hline Forward bending & 0.16 & 0.69 & 0.03 & 0.94 \\
\hline Leaning sideward & -0.10 & 0.98 & -0.51 & 0.16 \\
\hline Push-ups & -0.26 & 0.62 & 0.36 & 0.49 \\
\hline Electrical stimulation & 0.70 & $0.04^{a}$ & 0.54 & 0.14 \\
\hline
\end{tabular}

aSignificant correlation at $P<0.05$.

Table 4 Correlation of peak blood flow between different conditions

\begin{tabular}{lcr}
\hline & Peak blood flow & \\
\hline Correlation & Mean $( \pm$ s.d.) & P-value \\
\hline Rest- ES & $66.4(59.8)$ & 0.007 \\
Rest- bending Forward & $172.3(153.6)$ & 0.006 \\
ES- bending forward & $105.9(177.3)$ & 0.092 \\
\hline
\end{tabular}

*Significant correlation at $P<0.05$.

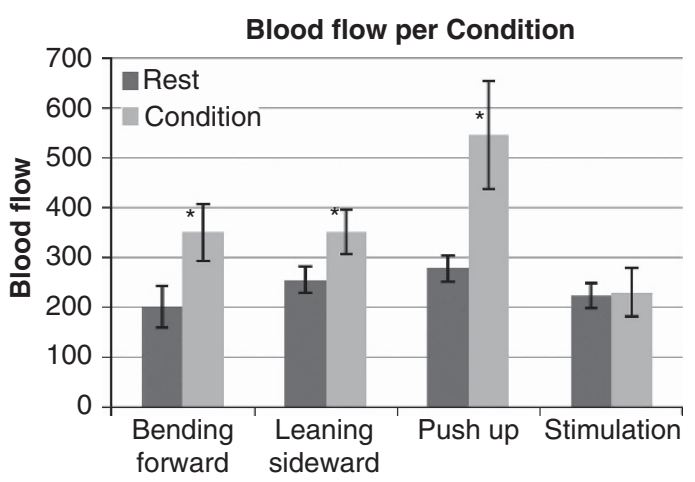

Condition

Figure 5 The mean BF under the tuber per condition, compared with rest just before performing the action. *Significant increase. 

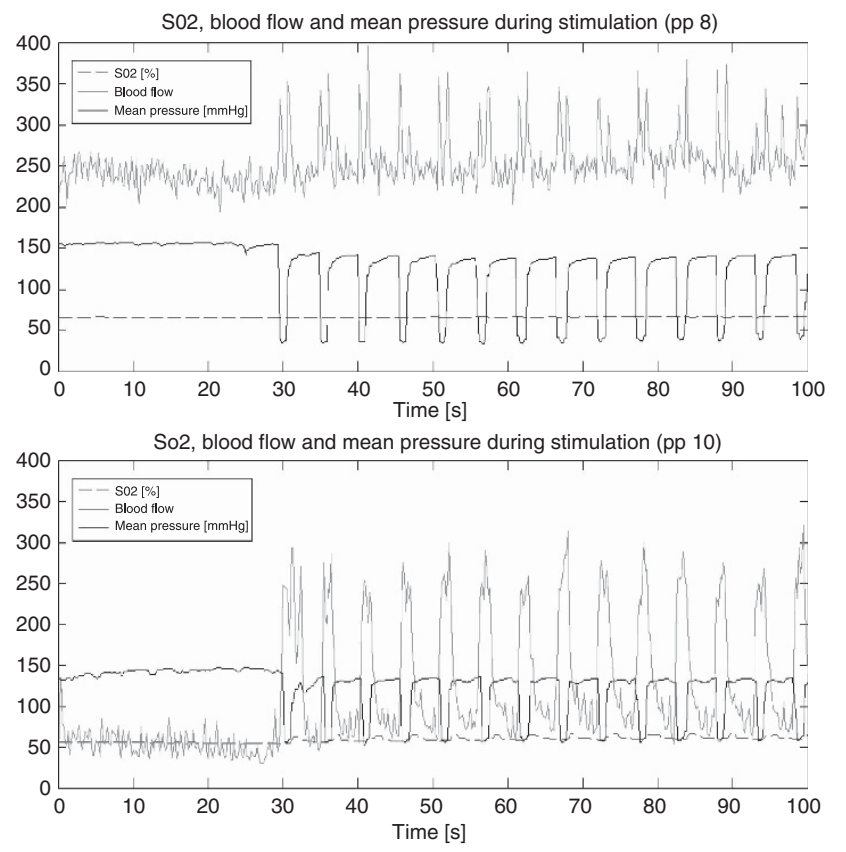

Figure $6 \mathrm{BF}$, oxygenation and mean pressure during rest and stimulation of two participants over time. ES started after $30 \mathrm{~s}$ of rest. Oxygenation is in $\% \mathrm{SO} 2$; mean pressure is in $\mathrm{mm} \mathrm{Hg}$; and $\mathrm{BF}$ is arbitrary and hence no unit is reported here.

course, but this significant difference in peak BF supports the finding in several studies that (prolonged) ES-induced muscle activation induces $\mathrm{BF}$.

Muscle activation did reduce IT pressure in eight participants, but the reduction may not have been adequate to increase mean BF and oxygenation. In all the four participants in whom no reduction of IT pressure was found due to muscle activation, more muscle atrophy was seen than in the other eight, possibly causing the different effect on IT pressure reduction due to (less absolute) muscle activation. In future studies, inducing stronger contractions with more current might be necessary, as well as an increasing sthe number of participants, for more statistical power.

Olive et al. ${ }^{15}$ reported impaired or altered BF to affected muscles in SCI patients during exercise or ES-induced contractions that may have contributed to increased muscle fatigue. ${ }^{15-17}$ Crespo Ruiz et al. ${ }^{13}$ described the physiological training effects: muscles will enlarge and become stronger and circulation will improve. ES-induced muscle activation with tetanic contractions allows mechanically unrestricted $\mathrm{BF}$ and may lead to less fatigue as a result of changes in tissue. Therefore, it is interesting not only to induce stronger contractions but also to enlarge the period of applying ES on gluteal and hamstring muscles. In the present study, peak BF is significantly increased by ES-induced muscle activation, and, although acute effects of ESinduced muscle activation may not be as good as those from PRMs, prolonged ES-induced activation and training of the gluteal and hamstring muscles may in contrast induce positive structural changes in muscle tissue, circulation and IT pressure distribution.

Further, ES-induced muscle activation in SCI results in improved cardio-respiratory capacity, such as maximal aerobic power, peak oxygen consumption, forced expiratory flow, and forced vital capacity. ${ }^{13,14}$ This may exert a decisive influence on the capacity to transport oxygen in the areas most affected by disuse resulting from the SCI itself. This is especially important when prolonged treatment is required, for example, in an attempt to prevent PUs. ${ }^{18}$ However, further studies are needed to confirm these findings.

There were two study limitations. One concerns the $\mathrm{O} 2 \mathrm{C}$ device, which is sensitive to disturbing influences. Although we followed the manufacturer's recommendations and carried out all measurements in the same room, with the same environmental factors, reduced amount of surrounding light and more or less constant temperature, the results can be influenced by the amount of light from the surrounding area and also by the temperature of the room. In three participants data were unreliable as the probe recorded too much surrounding light. The other limitation concerns the interface pressure mapping, which does not provide information about the load at deep tissue. Solis et al. found that superficial ES delivered every $10 \mathrm{~min}$ is sufficient to greatly reduce the extent of damage in the deep tissue exposed to constant external pressure. ${ }^{19,20}$ When the external load decreases, the internal load decreases as well, but more insight into the relationship between IT pressure and deep tissue injury is needed to provide information on the validity of external interface pressure measurements.

One of the clinical implications of this study relates to the effects of the different PRMs. Push-ups require good upper-limb strength and generate undesirable high pressure on the gleno-humeral joint, often resulting in shoulder injuries, whereas bending forward is easy to perform and does not place a heavy load on the shoulders. Our results confirm that bending forward and sidewards is as sufficient as a complete push-up for the recovery of the gluteal BF and oxygenation. For clinical (rehabilitation) practice, stronger evidence from the present study now confirms that push-ups should no longer be advised, but rather patients should be advised to bend forward to release IT pressure and improve gluteal BF and oxygenation.

\section{CONCLUSION}

Both PRMs and ES-induced muscle activation induced significant acute reductions in interface pressure. PRMs directly improved (sub)cutaneous oxygenation and peak and mean BF significantly, whereas ES-induced contractions increased peak BF and BF and oxygenation in some participants. Bending forward, leaning sidewards and push-ups showed similar increases in IT BF and oxygenation in SCI. The currently used ES method cannot replace PRMs, but it may be used additionally. ES-induced muscle activation is not as effective for acute pressure relief, but the frequency of muscle activation stimulation is much higher than the performance of PRMs and could therefore be more effective in the long term.

\section{DATA ARCHIVING}

There were no data to deposit.

\section{CONFLICT OF INTEREST}

The authors declare no conflict of interest.

1 Bogie KM, Wang X, Triolo RJ. Long-term prevention of pressure ulcers in high-risk patients: a single case study of the use of gluteal neuromuscular electric stimulation. Arch Phys Med Rehabil 2006; 87: 585-591.

2 Byrne DW, Salzberg CA. Major risk factors for pressure ulcers in the spinal cord disabled: a literature review. Spinal Cord 1996; 34: 255-263.

3 Gutierrez EM, Alm M, Hultling C, Saraste H. Measuring seating pressure, area, and asymmetry in persons with spinal cord injury. Eur Spine J 2004; 13: 374-379.

4 Bennett G, Dealey C, Posnett J. The cost of pressure ulcers in the UK. Age Ageing 2004; 33: 230-235.

5 Reenalda J, Van Geffen P, Nederhand M, Jannink M, IJzerman M, Rietman H. Analysis of healthy sitting behavior: interface pressure distribution and subcutaneous tissue oxygenation. J Rehabil Res Dev 2009; 46: 577-586. 
6 van Drongelen S, de Groot S, Veeger HE, Angenot EL, Dallmeijer AJ, Post MW et al. Upper extremity musculoskeletal pain during and after rehabilitation in wheelchair-using persons with a spinal cord injury. Spinal Cord Mar 2006; 44 : 152-159.

7 Ferguson ACB, Keating JF, Delargy MA, Andrews BJ. Reduction of seating pressure using FES in patients with spinal cord injury. A preliminary report. Paraplegia 1992; 30: $474-478$.

8 Levine SP, Kett RL, Cederna PS, Brooks SV. Electric muscle stimulation for pressure sore prevention: tissue shape variation. Arch Phys Med Rehabil 1990; 71: 210-215.

9 Levine SP, Kett RL, Gross MD, Wilson BA, Cederna PS. Blood Flow in the Gluteus Maximus of Seated Individuals During Electrical Muscle Stimulation. Arch Phys Med Rehabil 1990; 71: 682-686.

10 Liu LQ, Nicholson GP, Knight SL, Chelvarajah R, Gall A, Middleton FR et al. Interface pressure and cutaneous hemoglobin and oxygenation changes under ischial tuberosities during sacral nerve root stimulation in spinal cord injury. J Rehabil Res Dev 2006; 43: 553-564.

11 van Londen A, Herwegh M, van der Zee CH, Daffertshofer A, Smit CA, Niezen A et al. The effect of surface electric stimulation of the gluteal muscles on the interface pressure in seated people with spinal cord injury. Arch Phys Med Rehabil 2008; 89 1724-1732.

12 Smit CAJ, Haverkamp GLG, de Groot S, Stolwijk-Swuste JM, Janssen TWJ. Effects of electrical stimulation-induced gluteal versus gluteal and hamstring muscles activation on sitting pressure distribution in persons with a spinal cord injury. Spinal Cord 2012; 50: 590-594.

13 Crespo-Ruiz B, del-Ama AJ, Jiménez-Díaz FJ, Morgan J, de la Peña-González A Gil-Agudo ÁM. Physical activity and transcutaneous oxygen pressure in men with spinal cord injury. J Rehabil Res Dev 2012; 49: 913-924.

14 Rischbieth H, Jelbart M, Marshall R. Neuromuscular electrical stimulation keeps a tetraplegic subject in his chair. Spinal Cord 1998; 36: 443-445.

15 Olive JL, Dudley GA, McCully KK. Vascular remodeling after spinal cord injury. Med Sci Sports Exerc 2003; 35: 901-907.

16 Gerrits HL, de Haan A, Sargeant AJ, van Langen H, Hopman MT. Peripheral vascular changes after electrically stimulated cycle training in people with spinal cord injury. Arch Phys Med Rehabil 2001; 82: 832-839.

17 Kocina P. Body composition of spinal cord injured adults. Sports Med 1997; 23 : 48-60.

18 Janssen TWJ, de Koning A, Legemate KJA, Smit CAJ. Electrical stimulation-induced gluteal and hamstring muscle activation can reduce sitting pressure in individuals with a spinal cord injury: 658: May 27 3:45 PM-4:00 PM. Medicine \& Science in Sports \& Exercise 2009; 415: 41: 10.1249/1201.

19 Solis LR, Hallihan DP, Uwiera RR, Thompson RB, Pehowich ED, Mushahwar VK. Prevention of pressure-induced deep tissue injury using intermittent electrical stimulation. J App/ Physiol 2007; 102: 1992-2001.

20 McInnes E, Bell-Syer SE, Dumville JC, Legood R, Cullum NA. Support surfaces for pressure ulcer prevention. Cochrane Database Syst Rev 2008; 4: CD001735. 